Mots. Les langages du politique

Présidentielle 2007. Scènes de genre

\title{
La parole profane dans les médias ou les ambigüités du discours sur le genre
}

Aurélie Olivesi

\section{(2) OpenEdition \\ Journals}

Édition électronique

URL : https://journals.openedition.org/mots/19081

DOI : $10.4000 /$ mots. 19081

ISSN : 1960-6001

Éditeur

ENS Éditions

Édition imprimée

Date de publication : 1 juillet 2009

Pagination : 65-81

ISBN : 978-2-84788-151-6

ISSN : 0243-6450

Référence électronique

Aurélie Olivesi, « La parole profane dans les médias ou les ambigüités du discours sur le genre », Mots. Les langages du politique [En ligne], 90 | 2009, mis en ligne le 01 juillet 2011, consulté le 23 avril 2022. URL : http://journals.openedition.org/mots/19081; DOI : https://doi.org/10.4000/mots.19081 


\section{La parole profane dans les médias ou les ambigüités du discours sur le genre}

Le traitement de la campagne présidentielle de 2007 dans la presse écrite présente une particularité notoire: les principaux quotidiens français ont tous publié de longues séries de reportages dans lesquels sont mis en scène les propos de gens « ordinaires ». Aux 112 articles publiés en série dans Le Monde, La Croix, Libération, 20 Minutes, Le Parisien et Le Figaro peuvent être ajoutés des articles isolés, publiés dans la presse quotidienne et magazine'. L'ampleur de ce traitement suggère que ces reportages dépassent le cadre du simple micro-trottoir, mais occupent au contraire une fonction propre au sein du discours journalistique dans son ensemble².

Si la presse quotidienne utilise largement la citation du discours d'autrui pour construire son propre discours, le recours à une parole profane fait l'objet d'un usage particulier. Ce type de propos peut en effet être utilisé par un organe de presse pour "équilibrer» son discours et permettre ainsi aux journalistes de mettre en œuvre, au sein du discours journalistique, des arguments qu'ils reprennent tout en les mettant à distance3.

L'analyse de ce type de reportage se révèle particulièrement fructueuse pour étudier la place des représentations du genre (gender) dans l'élaboration du discours journalistique sur Ségolène Royal. En effet, l'usage de propos attribués aux gens «ordinaires» permet la mise en œuvre, à la marge, d'une

Université Paris 12 Val-de-Marne, aurelie.olivesi@wanadoo.fr

1. Voir tableau en annexe.

2. D'autant qu'on les trouve dans des rubriques prestigieuses: «Politique», "Présidentielle», «France». L'usage de ce type de reportage avait été bien moindre lors de l'élection présidentielle de 2002. On en trouve publiés de manière isolée dans La Croix, et en série dans Le Monde (Chronique «Carnet de campagne » en dernière page, du 6 au 20 avril 2002 ; pour l'étude de cette série, voir Restier-Melleray, 2005).

3. «Plutôt que d'assumer directement une position, [le locuteur journaliste] préfère mettre en scène un ensemble de voix, dont il se distancie ou avec lesquelles il se solidarise plus ou moins discrètement. » (Maingueneau, 2007, p. 112) C'est le cas notamment dans le courrier des lecteurs: «Critiqués pour leur positionnement», les magazines «veillent à équilibrer leur sélection, avec une légère surreprésentation» de l'opinion adverse. «Attaqués pour leur absence de neutralité, les hebdomadaires vont donner la parole à leurs détracteurs [...]. Les rédactions peuvent aussi mobiliser leurs lecteurs pour assurer la promotion d'un tel débat contradictoire, raisonné et détaché.» (Hubé, 2008, p. 7-8) 
représentation du genre en politique plus complexe et plus ambigüe que celle présente dans le discours dont les journalistes sont locuteurs.

\section{La délégation de la parole}

Les reportages de ce type peuvent être rattachés à un genre journalistique défini par Érik Neveu comme «journalisme ethnographique», dans lequel les journalistes font un « usage intensif de la citation, de la parole rapportée - et si possible colorée -, mais en contournant la forme "entretien", implicitement réservée à des interlocuteurs légitimes », et adoptent un "parti pris explicite de les narrativiser, de les "scénariser" "4. Ces reportages instaurent un rapport particulier entre discours citant et discours cité, que l'on peut qualifier de délégation de la parole. Cette délégation de la parole se fonde sur deux particularités énonciatives propres au discours rapporté dans la presse, qu’il convient d'expliciter afin d'analyser le rôle joué par ces propos rapportés de gens « ordinaires » dans la représentation du genre au sein du discours journalistique.

La première de ces caractéristiques est la prise de distance du journaliste par rapport aux propos qu'il cite. En effet, le locuteur journaliste, quand il «emprunte les mots d'un autre pour les introduire dans son propre discours», ne prend pas en charge "le contenu du message d'origine » (Komur, 2004, p. 61). Son interprétation est présente dans le cotexte du discours rapporté5. Cependant, dans ces séries de reportages, le discours citant intègre les propos rapportés dans sa propre linéarité, sans faire de commentaires métalinguistiques, comme cela est plus couramment le cas quand sont intégrés les propos de locuteurs seconds «légitimes», et non profanes. Ce «silence métalinguistique ${ }^{6}$ était déjà constaté par Christiane Restier-Melleray dans son étude consacrée aux «Carnets de campagne» publiés dans Le Monde en 2002. Elle signalait en effet que «l'illusion de transparence repose [...] sur l'art

4. Selon lui, ce «journalisme ethnographique» résulte du «souci de prendre mieux en compte ce [qui est perçu] comme l'attente d'une information plus concrète et proche du quotidien » : il s'agit de «textes courts», mettant «le plus souvent en scène des gens "ordinaires" " (Neveu, 2000, p. 191). La série publiée dans Le Monde en 2002 correspond à cette définition: "L'ensemble des Carnets repose sur l'utilisation des différentes palettes du discours rapporté avec un large recours aux dialogues, procédés donnant vie aux échanges d'arguments. [...] Ceci induit une impression de familiarité immédiate (c'est-à-dire sans intermédiaire apparent) avec les protagonistes des récits. » (Restier-Melleray, 2005, p. 70) II s'agit ainsi de « reproduire par la multiplicité des voix et des styles un scénario dialogal plus proche du vécu et donc plus récréatif que le monologue argumentatif» (Bastian, Hammer, 2004, p. 524).

5. «La relation entre la citation et le cotexte environnant est exemplaire, au sens que le cotexte assure une fonction explicitement métadiscursive et interprétative; il annonce et anticipe ce que la citation accomplit [...] ensuite. [...] C'est comme si le cotexte était une qualification au niveau métalinguistique de l'acte langagier qui vient après en forme de citation.» (Tuormala, 2004, p. 334) «Ce type de réflexivité métalinguistique permet d'exprimer l'attitude du journaliste, le plus souvent relevant de la distinction envers la séquence représentée. » (Komur, 2004, p. 61)

6. Selon l'expression de Simone Bonnafous, citée par Roselyne Koren (2001, p. 178). 
de l'intermédiaire, un journaliste narrateur dont la palette des prises de position va de l'effacement revendiqué [...] à la construction assumée de la mise en scène » (Restier-Melleray, 2005, p. 70). Le rapport entre discours citant et discours cité est également analysé par Sandrine Lévêque - lorsqu'elle étudie cet usage du reportage chez l' "homme de la rue» dans le traitement par la presse écrite du mouvement social de décembre 1995 - comme l'utilisation par les journalistes de "substituts à une analyse qui leur soit propre», par le biais de «dispositifs professionnels qui permettent aux journalistes d'asseoir la légitimité de leur parole sur celle de l'opinion publique [...], [mais] surtout de déléguer cette parole en gardant sur elle une certaine maîtrise »7. Ainsi, les paroles «qui sont retenues par les journalistes permettent aussi de déléguer une partie de l'interprétation [...] sur le modèle, "c'est pas nous qui le disons c'est eux" » (Lévêque, 1999, p. 104). C'est là «l'ambiguïté » fondamentale «de la prise de distance »: «le locuteur cité apparaît aussi bien comme le non-moi dont le locuteur se démarque » que comme l'« autorité qui protège l'assertion. On peut aussi bien dire "ce que j'énonce est vrai parce que ce n'est pas moi qui le dis" que le contraire» (Maingueneau, 1987, p. 61). Ulla Tuormala conclut ainsi que «la pratique journalistique de l'écriture exploite le DD [discours direct] pour rendre sa subjectivité moins apparente » (Tuomarla, 2000, p. 17).

La posture d'objectivité est la seconde caractéristique propre à l'emploi du discours rapporté dans la presse. La délégation de la parole permet de mettre au jour un discours extrêmement complexe et mouvant, dans ce que Roselyne Koren décrit comme une "oscillation entre deux pôles antithétiques» constitutive d'une «rhétorique de l'impartialité» propre au discours de presse (Koren, 2001, p. 178). Cette analyse rejoint le second volet d'interprétation suggéré par Sandrine Lévêque, selon laquelle «multiplier les "paroles" d'usagers [...] permet d'affirmer un certain pluralisme qui montre "l'objectivité" des rédactions» (Lévêque, 1999, p. 103-104).

Ainsi le discours journalistique peut-il fonder son objectivité en rapportant des propos divers, tout en les mettant à distance. Cependant, le fait qu'il les rapporte - c'est-à-dire qu'il se réserve la possibilité de les réécrire - sans les commenter, trace un rapport plus complexe entre discours citant et discours cité: le journaliste, qui fait à la fois usage et mention des propos des gens " ordinaires », les intègre au sein de sa propre argumentation, sans prendre en charge leur énonciation. Dès lors, on peut se demander dans quelle mesure cet enchâssement de discours ne fonctionne pas comme une légitimation

7. Le discours cité par la presse, et dont le journaliste n'est pas locuteur, n'en demeure pas moins un discours de presse: «À l'intérieur de la masse des discours que l'on trouve dans un journal [...], on rencontre aussi bien des textes produits à l'initiative de la rédaction [...] que des textes [...] qui, tout en étant publiés sous le contrôle de la rédaction, trouvent leur site de production initial en dehors de celle-ci, et impliquent des figures appartenant à d'autres composantes de l'espace public. [...] Le journal [...] apporte ensuite sa propre voix [...] en procédant à des coupes et à des réécritures. » (Krieg, 2000, p. 80-81) 
du discours cité qui se trouve, par ce phénomène de délégation de la parole, intégré au sein de l'espace public ${ }^{8}$.

Concernant la représentation du genre en politique dans la presse écrite au moment de l'élection présidentielle de 2007 , notre hypothèse est que le processus de délégation de la parole dans ce type de reportage va permettre la mise en œuvre de stéréotypes de genre absents du reste du discours journalistique et, de ce fait, l'élaboration d'un discours sur le genre plus complexe que celui qui prévaut dans le discours dont le journaliste est locuteur.

Le compte rendu médiatique de la campagne municipale de 2001, dans laquelle a été appliquée pour la première fois la loi sur la parité, a donné lieu à la construction d'une image du genre en politique fondée sur l'idée que les femmes auraient une approche différente du pouvoir9. Dans la mesure où ce discours ne tient pas compte des ressources politiques propres des candidates et met en avant leur identité féminine (il valorise l'entrée des femmes dans la sphère politique en valorisant leur position supposée de profane), il a été désigné par le terme de discours "profemme». Ce discours n'a pas été remis en cause entre 2001 et 2007 - notamment parce que la question du genre en politique a disparu du débat journalistique ${ }^{10}$. On verra ainsi, à travers l'étude des propos rapportés, que ce discours «profemme», qui semble incontesté, se révèle en réalité très diversement interprété.

\section{Classement des propos rapportés : la référence au genre de Ségolène Royal}

Afin de travailler sur un corpus clos et homogène, nous avons choisi d'étudier les propos rapportés sur Ségolène Royal dans les 112 reportages publiés sous

8. «Le journal quotidien est [...] devenu un substitut de l'espace public, un forum où l'on entend l'écho de toutes les voix publiques en même temps qu'il a sa propre voix. Cette dualité est à l'origine des stratégies par lesquelles il manipule, soit pour s'identifier à lui, soit pour s'en distancier, le discours d'autrui. » (Mouillaud, Tétu, 1989, p. 6)

9. «Les premières mises en œuvre de la loi sur la parité [...] furent l'occasion d'une surenchère autour de l'idée selon laquelle les femmes feraient de la politique différemment des hommes. » (Guionnet, 2002, p. 113)

10. "Le débat s'éteint du jour au lendemain, après le vote du Parlement. » (Fassin, 2002, p. 21) «Ce qui se dessine [...] durant ce premier scrutin paritaire c'est un nouveau registre de légitimation en politique, [...] que l'on pourrait nommer “profemme”, où l’identité de femme est indissociablement liée à celle de profane. » (Achin et al., 2007, p. 62) On trouve déjà ce terme chez Delphine Dulong et Sandrine Lévêque, selon qui, « durant toute la campagne, la presse ne cesse ainsi d'entretenir une confusion entre femmes et profanes. [...] Au point que l'on peut parler [...] de la constitution d'un registre de légitimation "profemme" " (Dulong, Lévêque, 2000, p. 103104). Catherine Achin considère de son côté que «l'argumentaire qui accompagne [la] candidature [de Ségolène Royal] est finalement assez comparable à celui des municipales de 2001 » (Achin et al., 2007, p. 165), même si l'auteure reconnait que «son succès aux primaires a été rendu possible tout d'abord par son propre capital politique» (ibid., p. 162). 
forme de séries ${ }^{11}$. On ne prendra donc pas en compte les articles isolés. On étudiera dans ce sous-corpus les différentes formes du discours rapporté en s'attachant particulièrement au discours direct et aux ilots textuels, sans perdre de vue la présence énonciative à l'intérieur même de ces propos, dans la mesure où ces derniers font l'objet d'une réécriture journalistique ${ }^{12}$. Nous prendrons ainsi en compte le cotexte des propos rapportés dans la mesure où il éclaire les enjeux de la représentation du genre au sein de la délégation de la parole.

Or, une lecture cursive de l'ensemble des propos rapportés sur tous les candidats révèle une particularité des propos portant sur Ségolène Royal: on rencontre 23 références explicites à son genre, ce qui n'est le cas pour aucun des autres candidats, hommes ou femmes. Cette spécificité nous amène à examiner en premier lieu ces paroles avant d'analyser les propos dans lesquels son genre n'est pas mentionné explicitement, et de montrer qu'il est néanmoins présent, de manière implicite. Il conviendra alors de mesurer l'écart entre les propos tenus sur le genre de manière explicite et ceux qui n'y font référence qu'implicitement.

\section{La référence explicite au genre de Ségolène Royal}

Dans le premier sous-corpus de travail, composé des propos où le genre féminin est évoqué de manière explicite, la répartition axiologique est notable: ces arguments sont, dans leur grande majorité (19 occurrences sur 23) utilisés de manière positive ("Je me dis "une femme, ça serait bien” » ou «La France a peut-être besoin d'une femme»); les quatre autres arguments ont une valeur neutre ("Le fait que ce soit une femme m'indiffère », ou «Qu'il s'agisse d'une femme ne m'influence pas »'13, alors que ces propos sont attribués à une femme qui se décrit comme de gauche et que "son histoire personnelle » aurait dû menervers la candidate socialiste). Il est particulièrement remarquable que les propos rapportés qui font explicitement référence au genre de Ségolène Royal ne le fassent jamais de manière négative.

En outre, trois thématiques se dégagent, que nous classerons en fonction de la cohérence thématique et lexicale des arguments développés. La

11. Voir note 2.

12. «Le triptyque canonique (DD, DI, DIL) [discours direct, discours indirect, discours indirect libre] est simplement trop artificiel et restreint pour couvrir nombre de phénomènes du DR [discours rapporté] [...]. La généralisation des discours énonciativement hétérogènes où le langage objet est réintégré et non enchâssé dans le discours citant est un phénomène frappant [du discours de presse]. » (Tuomarla, 2000, p. 146) Voir également Maingueneau, 2007, p. 114. Ainsi, dans la mesure où il s'agit d'une réécriture, même si certains types de propos sont cités plus ou moins abondamment, une analyse quantitative précise n'a pas lieu d'être.

13. Respectivement: F, Le Monde, 3 février; $\mathrm{H}$, Le Monde, 31 mars; F, Le Monde, 24 février; $\mathrm{F}$, Le Monde, 16 avril (mention F quand les propos sont tenus par une femme, H s'il s'agit d'un homme. Les journaux cités étant parus, sauf mention contraire, en 2007 , seuls le jour et le mois sont indiqués dans les références). 
première thématique s'articule autour de la valeur symbolique qu'aurait l'élection d'une femme à la présidence de la République : «Je veux une femme présidente dans ce pays qui a tellement l'image machiste » ou "Si c'est une femme qui est choisie, forcément, ça va donner l'exemple: il y aura plus de femmes aussi au gouvernement et au Parlement». Ce propos, prononcé par une jeune fille: «Aujourd'hui, les hommes acceptent de voir les femmes passer à la politique » ${ }^{14}$, souligne à la fois que le fait d'être une femme en politique est le fruit d'une lutte et représente un acquis récent, mais également que ce sont les hommes qui maitrisent le jeu politique.

La deuxième thématique reprend l'idée que la femme politique incarne en elle-même le changement, fût-il indéterminé. On retrouve de nombreuses occurrences de cette idée, exprimée en termes constants: "Pour que ça change, il faut une femme au pouvoir»/ «Une femme au pouvoir, cela va changer la donne, forcément» / «Une femme au pouvoir, ça peut aussi changer les choses» / «Peut-être que ça changera quelque chose, pour une fois, une femme » / "Lui aussi a l'intention de voter pour Ségolène Royal "parce que c'est une femme", "pour voir si ça peut changer les choses" ${ }^{15}$. Cette idée relève, comme on l'a vu, de l'une des thématiques principales du discours "profemme», qui prône un «réenchantement »16 de la politique par les femmes.

La troisième thématique, qui découle de la deuxième, véhicule quant à elle l'idée qu'une femme politique va pouvoir exprimer dans l'exercice de ses fonctions des valeurs typiquement «féminines». Cette thématique est plus diffuse, mais on y trouve des caractéristiques traditionnellement attribuées aux femmes. On relève l'attention à la famille (")e ne sais pas pourquoi mais je pense qu'une femme est plus à même de défendre l'écologie qu'un homme. Peut-être parce qu'elles sont plus soucieuses de l'avenir qu'on réserve aux enfants », "parce qu'elles comprennent mieux les jeunes et les scolaires», ou encore: «En tant que femme, elle comprend peut-être mieux la situation des familles»), mais également l'abnégation ("Une femme au pouvoir? Ça pourrait être pas mal, elle fera certainement passer l'intérêt général avant le sien »), et enfin le souci de l'ordre ( Une femme mettrait un peu d'ordre ${ }^{17}$ ). Les qualités attribuées aux femmes politiques sont ainsi celles d'une mère idéalisée, image «la plus banale» des femmes en politique (Sineau, 1988, p. 36-68), sans qu'on puisse établir de distinction en fonction du genre du locuteur,

14. Respectivement: $\mathrm{H}$, Le Monde, 20 janvier; $\mathrm{F}$, Le Monde, 27 janvier; $\mathrm{F}$, Le Parisien, 15 mars.

15. Respectivement: F, 20 Minutes, 22 mars; F, Le Monde, 16 avril ; H et F, Le Monde, 30 décembre 2006 ; $\mathrm{H}$, Le Monde, 17 février; $\mathrm{H}$, Le Monde, 17 février.

16. «[L]es discours dominants de la parité [...] justifient l'entrée des femmes en politique par leur capacité à faire de la politique autrement et "réenchanter" la vie politique en y introduisant des valeurs réputées féminines, telles la douceur, le désintéressement, la "concrétude”, etc. » (Achin, Lévêque, 2007, p. 33) Voir également Achin, Dorlin, 2007 et Achin et al., 2007, p. 38.

17. Respectivement: F, La Croix, 27 avril ; F, La Croix, 13 avril ; F, Le Parisien, 22 février; H, Libération, 2 février; $\mathrm{H}$, Le Monde, 24 mars. 
puisque les propos sont prononcés dans les mêmes proportions (12 femmes, 10 hommes et un couple) par des locuteurs masculins et féminins ${ }^{18}$.

On peut ainsi distinguer dans les propos rapportés faisant explicitement référence au genre de Ségolène Royal un phénomène de «retournement du stigmate» (Achin et al., 2007, p. 62 ; Dulong, Matonti, 2005, p. 298), identique à celui présent dans le discours "profemme»: l'identité de genre se trouve érigée en ressource politique. Le discours issu de la campagne municipale de 2001, fondé sur l'idée que les femmes apporteraient une plus-value à la politique, semble donc toujours présent. Cependant, les propos cités dans ces séries de reportages ne font pas tous explicitement référence au genre de Ségolène Royal. Il convient donc d'examiner si cette référence ne demeure pas toutefois présente, mais de manière implicite et, le cas échéant, d'en interroger la valeur axiologique.

\section{La référence implicite au genre de Ségolène Royal}

On retrouve, dans les propos rapportés qui portent sur Ségolène Royal sans faire référence explicitement à son genre (il s'agit de notre second souscorpus de travail), des thématiques qui recoupent celles présentes dans les propos qui y font référence explicitement. Il convient donc de s’interroger sur une possible référence implicite au genre de Ségolène Royal.

\section{L’incarnation de la nouveauté en politique}

On retrouve en premier lieu, dans les propos qui portent sur Ségolène Royal sans référence explicite à son genre, la deuxième thématique que nous avions dégagée dans les propos qui y faisaient explicitement référence : celle de l'espoir dans le changement incarné par la candidate ("Ségolène Royal apportait de la nouveauté, une autre vision des choses» / «L'arrivée de Ségolène Royal m'a donné l'espoir qu'on pouvait être au pouvoir différemment» / "J'espère qu'elle va changer les choses »). Ce changement est par ailleurs assimilé à une forme de modernité («Elle est moderne et novatrice» / "Ségolène incarne à fond le $x x I^{e}$ siècle ${ }^{19}$ ).

18. De la même manière que l'identité de genre, l'identité socioprofessionnelle des locuteurs ne constitue pas un critère pertinent de classement des arguments. La raison en est toutefois différente: les articles de «journalisme ethnographique» interrogent des catégories socioprofessionnelles relativement constantes d'un organe de presse à l'autre (agriculteurs, pêcheurs, chômeurs, mineurs, ouvriers, petits propriétaires, personnes vivant à l'année dans des campings...), et souvent éloignées sociologiquement du lectorat du journal - même s'il faut souligner l'exception du Figaro, qui a également donné la parole à des catégories socioprofessionnelles plus proches de son lectorat (médecins, militaires, patrons).

19. Respectivement: $\mathrm{H}$, Le Monde, 13 avril; $\mathrm{H}$, Libération, 2 février; $\mathrm{H}$, Le Parisien, 19 avril; $\mathrm{H}$, Le Monde, 20 janvier; $\mathrm{H}$, Le Monde, 20 janvier. 
Cependant, si la nouveauté qu'incarne Ségolène Royal est évaluée positivement, elle ne l'est pas de manière absolue, mais en référence aux hommes du PS. L'idée selon laquelle Ségolène Royal serait affranchie des rigidités du fonctionnement institutionnel du Parti socialiste recoupe une des thématiques du discours «profemme». Face aux «éléphants» du PS, Ségolène Royal est, dans les propos rapportés, placée dans une position active puisqu'elle est présentée comme les «bousculant» ou "s'opposant» à eux. L'attitude de Ségolène Royal est présentée, dans un énoncé négatif, comme le contraire de celle des "éléphants» («Elle n'a pas d'œillères, ne donne pas le sentiment de détenir la vérité absolue»), ou bien dans un énoncé comparatif, en référence à eux («Elle est plus ouverte, moins sclérosée que le reste du PS »20). Cette construction est souvent relevée dans la représentation des femmes politiques: elles sont qualifiées par rapport aux hommes. On retrouve ces comparatifs dans ce propos décrivant Ségolène Royal comme «plus à l'écoute, pragmatique, consensuelle ${ }^{21}$. La conversion du genre en ressource politique en fait toutefois une ressource relative et, par conséquent, fragile, comme le souligne l'analyse des propos portant sur la personnalité de la candidate.

\section{Une personnalité perçue comme sans envergure}

Quand on évoque la personnalité de Ségolène Royal, on est confronté au phénomène de versatilité axiologique des représentations relevé par JeanPaul Honoré à propos de la représentation du Japon dans la presse: le lecteur est «confront[é], sur le plan axiologique, à deux réseaux d'images, les unes favorables et les autres critiques [qui] participent [cependant] d'un seul et même système. [...] On retrouve donc des stéréotypes identiques sous des hypostases différentes, au cœur de formations discursives qui s'opposent superficiellement» (Honoré, 1994, p. 10). C'est notamment dans les propos évoquant la démocratie participative que l'on observe un basculement qui se révèle éclairant pour voir comment la qualification positive de l'ouverture d'esprit de Ségolène Royal peut se muer en un portrait à charge. Ainsi, à l'opposé d'une opinion favorable à cette pratique politique («Je crois à la démocratie participative. Même s’il ne s'exprime pas forcément très bien, le peuple a des choses à dire»), on trouve des propos qui critiquent «la démagogie de

20. Respectivement: $\mathrm{H}$, Le Monde, 13 avril; $\mathrm{H}$, Le Monde, 31 mars; $\mathrm{H}$, Le Monde, 16 avril; $\mathrm{H}$, Le Monde, 21 avril.

21. H, Libération, 30 mars. On retrouve les éléments constitutifs du discours «profemme» tel qu'il a été décrit par Christine Guionnet: "un plus grand pragmatisme, un rapport moins ambitieux, moins carriériste à la politique [...], une volonté d'être plus efficaces [...], une plus grande faculté à entretenir des relations de proximité avec les autres [...] et à demeurer à l'écoute de leurs interlocuteurs, une appétence singulière pour les domaines sociaux [...] et, a contrario, un moindre intérêt pour les considérations partisanes, les dossiers techniques, les prises de parole et les relations violentes et conflictuelles, etc. » (Guionnet, 2002, p. 114). 
la démocratie participative» et sous-entendent que cette pratique est révélatrice d'une absence d'idées («Impossible de prendre au sérieux quelqu'un qui fonde son programme en disant aux gens que l'on fera ce qu'ils veulent» / «Elle, elle attend que les citoyens lui donnent des idées» / "On ne sait pas ce qu'elle pense» / «Ségolène on ne voit pas très bien sa ligne »). Ce qui était considéré comme une preuve d'ouverture d'esprit se transforme en «manque de rigueur» ou manque de cohérence ("C'était plus cohérent que Ségolène ») qui se caractérise par un «flou » ("Ségolène reste un peu floue »²2).

Ce «flou» est également censé s'exprimer à travers une inconséquence verbale présumée: on trouve des propos évoquant l'idée que Ségolène Royal multiplie les promesses ("si Ségolène fait tout ce qu'elle a promis, elle ne va pas s'en sortir et le pays non plus») et les déclarations contradictoires, sans se soucier de son parti («Ségolène Royal donne le sentiment d'être déconnectée de son propre parti, de faire cavalier seul; or, on ne peut pas être intelligent en avançant sans personne derrière soi») ou de son électorat («Royal a réussi le tour de force de se mettre son principal électorat, les profs, à dos, avec ses déclarations sur les 35 heures»). Ce «flou» et l'inconséquence verbale qui le caractériserait sont également interprétés comme un manque d'assurance ("Elle me donne le sentiment de ne pas être vraiment sûre d'elle et ses propositions gagneraient à devenir plus concrètes 》 / «Je la sens fragile») qui lui-même devient de la «mollesse» ("Ségolène est molle, son élocution est molle» / «... et Ségo un peu molle»). Ce dernier qualificatif fait mention d'une faiblesse à la fois physique, psychologique, politique (que l'on retrouve dans l'évocation du «consensus mou») et morale. Cette faiblesse supposée la rendrait inapte à la conduite des affaires ("Je la sens un peu petite pour la fonction, mais il y a beaucoup de gens bien autour d'elle» / «Elle se fait manipuler, elle ne peut pas être présidente» / «Royal est trop influençable, elle ne pourra rien faire malgré toute sa bonne volonté»), mais la place en position d'exécutante. Un propos tel que "Ségolène, ça serait juste une bonne Première ministre » rejoint les interrogations du type "est-ce que c'est vraiment elle qui va diriger?» L'idée qui transparait est que «Ségolène Royal n'a pas la stature ${ }^{23}$ pour être présidente.

Ce retournement axiologique souligne l'ambigüité de l'usage du genre dans les représentations politiques. Comme Catherine Achin et Marion Paoletti en avaient fait l'hypothèse, les femmes politiques qui jouent du retournement du stigmate en mettant leurs qualités féminines en avant se trouvent

22. Respectivement: $\mathrm{H}$, Le Monde, 16 avril ; F, Le Monde, 26 avril; ibid.; $\mathrm{H}$, Le Parisien, 8 mars; $\mathrm{H}$, Libération, 15 janvier; $\mathrm{F}$, Le Monde, 17 mars ; F, Le Monde, 26 avril ; $\mathrm{H}$, Le Monde, 13 avril ; $\mathrm{H}$, Le Parisien, $1^{\text {er mars. }}$

23. Respectivement: F, La Croix, 3 avril ; F, Le Monde, 26 avril ; F, Le Parisien, 13 avril ; F, Le Monde, 16 avril ; F, La Croix, 19 avril ; F, La Croix, 30 mars; F, Le Monde, 17 mars; $\mathrm{H}$, Le Monde, 12 avril; F, La Croix, 10 avril ; H, La Croix, 26 avril; ibid.; F, Le Monde, 17 mars; H, Libération, 2 février; $\mathrm{H}$, La Croix, 26 avril. 
valorisées dans un premier temps mais, en ne contredisant pas les représentations sociales les plus traditionnelles, qui les placent en position d'altérité par rapport au monde politique, elles se voient ensuite confinées dans des positions secondaires. C'est ce qu'elles ont nommé le «salto du stigmate» (Achin, Paoletti, 2002, p. 54). C'est à cette position dominée que l'on pourrait rapporter la référence au sourire de Ségolène Royal.

\section{L’interprétation du sourire de Ségolène Royal}

L'ambigüité du sourire des femmes politiques a été interprétée par Jane Freedman à partir des analyses d'Erving Goffman. Selon ce dernier, le sourire sert aux femmes d' "adoucisseur rituel » afin de faire excuser leur présence dans un monde qui n'est pas le leur (Goffman, 1988, p. 43, cité par Freedman, 1997, p. 86-87). Or, l'interprétation du sourire de Ségolène Royal dans les propos rapportés est différente: son sourire est interprété comme un signe d'infériorité ou de conformisme («Elle a toujours le même sourire un peu figé. Elle me donne l'impression de celle qui a toujours bien fait ses devoirs, mais cela ne suffit pas »24), ou comme une façade séduisante: «Elle, c'est toujours “cheese”, comme si tout allait bien. Moi, j’aime pas les séductrices. »25

Mais ce sourire relève également d'une hexis corporelle assez distante, qui révèle une ambigüité entre image sociale et image de genre ${ }^{26}$. En effet, cette distance qu'elle met en place, qu'on peut interpréter comme de la réserve ${ }^{27}$, est assimilée à une hexis distante de bourgeoise, comme on le voit dans un propos qui place sur le même plan « grands sourires et beaux tailleurs ${ }^{28}$. On retrouve à propos de Ségolène Royal (et non à propos des candidats masculins, même s’il s'agit d'un reproche fait aux «politiques» en général29), des

24. F, La Croix, 28 mars.

25. H, Le Monde, 17 février. On retrouve dans ces propos le rapport des femmes politiques à la séduction souligné par Jane Freedman: «L'idée de la séduction est l'idée du contre-pouvoir féminin par excellence [...] caractérisé comme un pouvoir secret qui détourne l'ordre établi. [...] La séduction porte toujours une signification de vice.» (Freedman, 1997, p. 122-123)

26. «Cette [...] réticence à se livrer peut être lue dans l'interdiction formelle faite par la candidate aux journalistes de la filmer en train de manger : consigne symptomatique du refus de montrer son corps dans des fonctions de base [...], et qui renvoie à une forme d'interdit face au corps partagé par les classes bourgeoises. Ségolène Royal reste ainsi une "icône”, selon la formule maintes fois employée par les médias pour la désigner. » (Coulomb-Gully, 2009)

27. "Lorsque [une] réunion [mixte] comprend des hommes autres que son mari», la femme est incitée «à observer son quant-à-soi. [...] Toute hâte de sa part, toute initiative, toute attitude excessive, toute agressivité [...] peut être considérée comme une invite sexuelle, bref, un signe d'accessibilité» (Goffman, 2002, p. 71).

28. F, Libération, 2 février.

29. Quand on recense les propos rapportés sur les hommes politiques en général, se dégage très nettement une thématique sur leur isolement par rapport à la population : «Ces gens-là ne roulent pas pour nous, les petits» (20 Minutes, 2 avril); "Aveugles, sourds, les hommes politiques ne connaissent pas leurs situations, leurs difficultés, répètent-ils. Trop éloignés du peuple» (Le Monde, 23 décembre 2006); "Ce ne sont pas des gens comme nous, ils sont dans leur monde» (La Croix, 6 avril); «Je me demande si les candidats tiennent compte des familles les 
critiques sur le fait qu'elle est «trop éloignée du peuple, des gens comme nous »30. On lit ainsi : «Elle n'est pas assez proche des préoccupations des Français. J'aimerais lui filer 1 ooo euros et qu'elle me dise comment on fait pour vivre avec! $3^{1}$, ou «Je ne sais pas si elle peut comprendre les pauvres

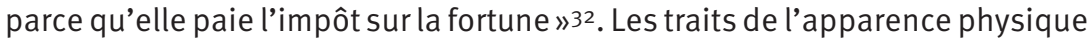
de Ségolène Royal qui pourraient relever de la séduction (sourire, élégance, réserve) sont interprétés négativement, comme de la distance. Les propos rapportés lui attribuent des caractéristiques féminines qui, sans être pour autant identifiées comme telles, sont évaluées négativement. Cette caractérisation se retrouve dans les propos évoquant ses propositions politiques.

\section{Ses options politiques présumées : spécialisation dans le social et incompétence sur le plan international}

Les options politiques présumées de Ségolène Royal dans les propos rapportés rejoignent largement les «rôles» stéréotypés décrits par Mariette Sineau qui évoque «la recréation "spontanée” de la séculaire division entre le féminin/social et le masculin/politique»33: les femmes politiques sont décrites comme ayant une appétence particulière pour le domaine social, qui a pour corollaire une incapacité dans les domaines prestigieux : économie et politique internationale.

\section{La spécialisation dans le social}

Les propositions en politique intérieure de Ségolène Royal citées dans les propos rapportés peuvent être regroupées sous une thématique unique, le social34, et à une proposition quasi exclusive: le SMIC à 1500 euros. Il est à noter qu'à une exception près35, cette proposition rencontre un écho négatif

plus démunies et s’ils connaissent leur situation » (La Croix, 13 avril); « Les politiques sont toujours en décalage par rapport à la réalité » (Le Figaro, 13 mars); "Les hommes politiques sont dans leur bulle. Ils sont loin de nos vies » (La Croix, 27 avril); "Il y a un décalage profond entre les politiques et le terrain» (Le Parisien, 29 mars). On peut noter que ce reproche n'est jamais formulé à propos de Nicolas Sarkozy dans les articles de notre corpus.

30. H, La Croix, 6 avril.

31. H, Le Parisien, 29 mars. Le même rattache ainsi Ségolène Royal à la classe politique dans son ensemble: "Ça vaut pour elle, mais ça vaut aussi pour les autres. »

32. F, La Croix, 19 avril.

33. «Division sous-tendant la primauté du politique par rapport au social, et la subordination du second par rapport au premier. » (Sineau, 1988, p. 41)

34. L'attribution d'un intérêt spécifique pour une politique sociale pourrait résulter de la prise en compte de son appartenance au Parti socialiste. Cependant, nous n'avons trouvé aucune occurrence de la thématique du «social» dans les propos rapportés concernant Lionel Jospin dans les «Carnets de campagne» publiés par Le Monde en 2002. Cette hypothèse semble donc invalide.

35. H, Le Parisien, 19 avril. 
("Il y a quand même une chose avec laquelle je ne suis pas d'accord; le smic à 1500 euros»), puisque l'on considère que cette proposition n'est pas crédible, «manque de sérieux» ou qu'il s'agit de «poudre aux yeux»36.

Les autres propositions (le «service public de la caution » et «la volonté de généraliser la culture artistique dès l'école») la ramènent à son statut symbolique de mère de famille, de la même manière que la troisième thématique que nous avions dégagée dans notre premier sous-corpus. Cette fonction maternante est même évoquée explicitement, dans les propos d'un jeune homme interrogé: "Ségo, elle est comme ma mère.» Le fait que le social constitue, comme on l'a vu, un domaine d'action politique globalement dévolu aux femmes est confirmé par un propos évoquant une fonction plus appropriée pour Ségolène Royal: "Elle ferait sans doute un très bon ministre des Affaires sociales et familiales. $» 37$

Comme le rappelle Mariette Sineau, la «spécialisation forcée» (Sineau, 1988, p. 42) des femmes dans le social ne relève pas tant d'une fonction maternante traditionnellement attribuée au genre féminin que d'une dévaluation de ces fonctions par rapport aux fonctions les plus prestigieuses, et qui appartiennent en propre au chef de l'État. Le «social» est dévalué dans le discours rapporté: introduit par la négation exceptive ne que («l'ai compris qu'elle ne ferait que du social »38), intensifié par l'adverbe trop («Royal, elle, est trop dans le social »39), qualifié, avec des réserves, de «bien joli », où la valeur concessive de l'adverbe bien est renforcée par la conjonction mais («Royal, elle veut faire du social, c'est bien joli, mais... »40), le social est considéré comme un domaine utile, mais peu prestigieux. Ainsi, l’image de la compétence dans ces fonctions dévaluées du social a pour corollaire une image d'incompétence dans les fonctions prestigieuses.

\section{L'incompétence présumée dans les domaines prestigieux}

En politique intérieure, sa compétence supposée pour le social peut être rapportée à son incompétence supposée en économie41. On en trouve trois

36. Respectivement: $\mathrm{H}$, Le Monde, 16 avril; $\mathrm{H}$ et F, 20 Minutes, 5 avril ; F, Le Monde, 26 avril; $\mathrm{H}$, Libération, 9 mars.

37. Respectivement: H, 20 Minutes, 4 avril; H et F, Le Parisien, 1 er mars; H, Libération, 16 mars; F, Le Monde, 3 février.

38. F, La Croix, 2 avril. La négation exceptive « est restrictive en ce qu'elle exclut de son champ tout terme autre que celui qu'elle introduit. On peut expliciter cette signification en ajoutant l'expression et rien d'autre» (Pellat, Riegel, Rioul,1994, p. 412-413).

39. H, Le Monde, 14 avril. Cet adverbe marque "une intensité dépassant une norme» (Pellat, Riegel, Rioul, 1994, p. 363).

40. F, Le Monde, 10 février.

41. "Dans le discours de la presse, ces “dames de cœur”, bien qu'elles soient habiles à s'occuper des problèmes sociaux [...], passent souvent pour utiliser plus d'intuition que d'intelligence dans leur travail [...]. Un autre constat [...] est qu'elles sont perçues comme complètement ignorantes en ce qui concerne l'économie.» (Freedman, 1997, p. 168-172) 
occurrences: deux provenant de chefs d'entreprise qui mettent en doute sa compétence (")'ai le sentiment que Ségolène Royal n'a pas bien compris comment fonctionne une entreprise », ou «Sans création de richesses, il n'y a rien à redistribuer. Ségolène Royal paraît loin de ces réalités, ignorante de ces sujets»), la troisième provenant d'un professeur d'économie qui considère sa «faiblesse en économie» comme un acquis ("l'ai rejeté Ségolène pour plein de petits points, mais surtout pour sa faiblesse en économie »42). La caractérisation socioprofessionnelle des locuteurs présente ici l'intérêt d'accentuer le poids du propos en donnant aux personnes interrogées une posture d'experts. La faiblesse supposée en économie va de pair avec une incompétence supposée concernant les relations internationales43.

Ce point est important dans la mesure où le voyage à l'étranger constitue un moment clé de la construction de la stature du candidat à la présidentielle44. La critique se concentre principalement sur son voyage en Chine, perçu dans le discours rapporté comme une promenade inutile (rapportée sous forme de question: «À quoi ça sert d'aller en Chine?») ou bien un facteur de risque ("Si elle passe, on frôlera sans cesse la catastrophe, comme dans ses voyages en Chine ou au Moyen-Orient! »). Ce voyage en Chine cristallise chez certains le rejet ou l'incompréhension qu'inspire la candidate: " "Hélène Ségara, elle veut faire bosser les Chinois." Heu, Ségolène, vous voulez dire? "Ségara, Ségolène, c'est pareil." » 45 La référence à une chanteuse populaire souligne une focalisation sur le physique de Ségolène Royal, ainsi qu'un déni de sa capacité à résoudre le problème considéré comme principal : l'emploi.

À ce premier volet de critiques se greffe le déni de sa capacité à représenter le pays ("Ségolène ne m'attire pas, je n'arrive pas à l'imaginer représenter la France»). À l'inverse, lorsque l'un des propos cités lui attribue les qualités nécessaires à un rôle international, il n'est pas question de stature. En effet, un chef d'entreprise livre le propos suivant: «Comme présidente, elle sera une excellente VRP politique pour vendre nos technologies à l'étranger. » En faisant de la candidate une «VRP», il lui attribue une simple fonction commerciale, et non un réel pouvoir de direction sur la politique étrangère. On retrouve, dans cette image d'incompétence dans le domaine de la politique internationale, au-delà de ses faiblesses personnelles supposées ("à l'international, elle ne tiendra pas le coup»), les constructions discursives qui servent traditionnellement à qualifier les femmes politiques, dans la mesure

42. Respectivement: H, Le Figaro, 17 mars; H, Libération, 30 mars; H, La Croix, 29 mars.

43. Il s'agit également d'une constante dans le domaine de la représentation des femmes politiques, «accusées [...] de manquer de compétence économique ou diplomatique : elles agissent précipitamment, ne réfléchissent pas avant de parler» (Freedman,1997, p. 167).

44. Christian Le Bart voit notamment dans le voyage à l'étranger une manière de conquérir la légitimité politique «d'en haut, celle, classique, du visionnaire ou de l'homme d'État, légitimité qui autorise à parler en surplomb de la société et du monde-comme-il-va » (Le Bart, 2008, p. 3).

45. Respectivement: H, 20 Minutes, 6 avril ; F, Le Monde, 20 janvier ; H, Libération, 2 février. 
où la seule opinion sur la politique internationale attribuée de manière positive à Ségolène Royal, l'est dans une phrase construite négativement («Au moins elle n'est pas atlantiste comme Sarko »46), et relativement aux opinions de Nicolas Sarkozy 47 , alors que le projet de politique internationale de ce dernier n'est jamais évoqué dans les propos rapportés.

Ainsi, lorsque l'on compare les propos rapportés mentionnant explicitement le genre de Ségolène Royal et ceux qui n'y font référence que de manière implicite, on s'aperçoit que, si le genre féminin parait être un atout en politique dans les propos où il est mentionné explicitement, les références implicites se trouvent en revanche négativement connotées. La menace d'un «salto du stigmate » évoquée par Catherine Achin et Marion Paoletti se trouve bien mise en œuvre dans le discours rapporté sur la candidature de Ségolène Royal dans les reportages chez les «gens "ordinaires" »: évoqué implicitement, le genre féminin est perçu comme un atout dans les fonctions subalternes et une entrave pour l'accès aux postes politiques prestigieux 48 .

L'usage de la délégation de la parole dans les reportages appartenant au genre du «journalisme ethnographique» intègre au discours journalistique des propos dont les journalistes ne prennent pas en charge l'énonciation. Est ainsi mise en œuvre, à la marge du discours journalistique, une représentation du genre particulièrement complexe, puisque ce discours se révèle luimême à double ressort: le genre y est évoqué explicitement de manière positive, et implicitement de manière négative.

La complexité des représentations est en outre matérialisée par l'organisation de ces reportages selon le principe de l'hyperstructure49. En juxtaposant les courts témoignages, au sein de la même "aire spatiale» (Mouillaud, Têtu, 1989, p. 5) de la page, ou en intégrant ces propos aux analyses politiques, le journal peut complexifier son discours afin de présenter le plus de facettes possibles d'un même phénomène, sans se risquer à en livrer une interprétation.

Le «silence métalinguistique» qui entoure ces propos «ne prouve pas [pour autant] que [le journaliste rapporteur] a renoncé à toute forme d'engagement et d'adresse à autrui » (Koren, 2001, p. 181). Au contraire, comme le rappellent Roselyne Koren et Alain Rabatel, « les journalistes [...] sont exposés

46. Respectivement: F, Le Monde, 24 février; $\mathrm{H}$, Le Parisien, 26 avril ; F, Le Monde, 3 février; $\mathrm{H}$, Le Monde, 20 janvier.

47. On trouve toutefois un parallèle entre genre et stature internationale: «L'homme politique idéal doit être - si j’ose dire - “couillu”, surtout pour ce qui concerne les relations internationales. Même si par moments, il semble difficilement contrôlable, je ne déteste pas le côté Napoléon, fonceur, peur-de-rien de Nicolas Sarkozy. » (F, Le Monde, 14 avril 2007)

48. Évoquant l'évolution des stéréotypes de genre, Ruth Amossy suggère ainsi que les femmes sont tombées «de Charybde en Scylla (Amossy, 1991, p. 172).

49. «Ensemble rédactionnel [...] formé d'un ensemble d'articles et d'images graphiquement regroupés et complémentaires, bornés à la limite matérielle de l'aire scripturale vi-lisible de la double page. » (Lugrin, 2001, p. 7-12) 
à l'expression de termes, de points de vue circulants qu'ils reprennent sans pouvoir toujours les mettre à distance». Selon eux, «l'effacement énonciatif règne en maître» dans le cas de la reprise de "termes ou [...] cadres d'analyse qui engagent des représentations du monde naturalisées», comme l'est le discours sur les femmes issu du vote de la loi sur la parité, qui est l'héritier des thèses féministes essentialistes50. Ils se demandent donc si «faire écho à ces manières de voir, sans s'en distancier (au nom de l'objectivité) ne participe [...] pas, fût-ce indirectement, de la reconduction de manières de voir et de penser qui mériteraient par ailleurs d'être discutées» (Koren, Rabatel, 2008, p. 16). Ainsi, l'usage des propos rapportés des gens «ordinaires» portant sur la candidature de Ségolène Royal permet de légitimer la présence au sein de l'espace public d'un discours sur le genre en politique fort différent de celui dont les journalistes prennent eux-mêmes en charge l'énonciation.

\section{Références}

ACHIN Catherine et al., 2007, Sexes, genre et politique, Paris, Economica.

ACHIN Catherine, Dorlin Elsa, 2007, «"J'ai changé, toi non plus”. La fabrique d'un-e présidentiable: Sarkozy/Royal au prisme du genre», Mouvements [en ligne] [URL: http://www.mouvements.info/spip.php?article42], site consulté le 3 mars 2009.

ACHIN Catherine, LÉVÊQUE Sandrine, 2007, «Femmes, énarques et professionnelles de la politique. Des carrières exceptionnelles sous contraintes", Genèses, n 67 , p. 22-44.

ACHIn Catherine, PAOlettI Marion, 2002, «Genre et construction des listes aux municipales de 2001. Le salto du stigmate », Politix, $\mathrm{n}^{\circ} 60$, p. 33-54.

Amossy Ruth, 1991, Les idées reçues. Sémiologie du stéréotype, Paris, Nathan.

BAStIAn Sabine, Hammer Françoise, 2004, "La citation journalistique. Une étude contrastive", Le discours rapporté dans tous ses états, J. M. Munoz, S. Marnette, L. Rosier éd., Paris, L'Harmattan, p. 519-528.

Coulomb-Gully Marlène, 2009, «Le corps présidentiel. Représentation politique et incarnation dans la campagne présidentielle française de $2007 »$, Mots. Les langages du politique, n89, 2007. Débats pour l'Élysée.

Dulong Delphine, LÉVÊQUE Sandrine, 2000, «Une ressource contingente. Les conditions de reconversion du genre en ressource politique », Politix, $n^{\circ}$ 6o, p. 81-111.

Dulong Delphine, Matontı Frédérique, 2005, «L'indépassable "féminité". La mise en récit des femmes en campagne », Mobilisations électorales. À propos des élections municipales de 2001, J. Lagroye, P. Lehingue, F. Sawicki éd., Paris, PUF, p. 281-303.

FASSIN Éric, 2002, "La parité sans théorie. Retour sur un débat», Politix, n 60, p. $19-32$.

50. «La collusion politique a neutralisé l'enjeu hautement symbolique et politique de la parité [...]: rendre légitime une approche essentialiste des rapports entre les sexes dans la société française.» (Lagavre, 2000, p. 126) 
Freedman Jane, 1997, Femmes politiques. Mythes et symboles, Paris, L'Harmattan. GoffmAn Erving, 1988, Les moments et leurs hommes, Paris, Le Seuil.

- 2002, L'arrangement des sexes, Paris, La Dispute.

GUIONNET Christine, 2002, «Entrées de femmes en politique. L'irréductibilité du genre à l'heure de la parité», Politix, n 60, p. 113-146.

HONORÉ Jean-Paul, 1994, «De la nippophilie à la nippophobie. Les stéréotypes versatiles dans la vulgate de presse (1980-1993) », Mots. Les langages du politique, $\mathrm{n}^{\circ}{ }_{41}$, Parlerdu Japon, p. 9-55.

Hubé Nicolas, 2008, «Le courrier des lecteurs. Une parole journalistique profane?», Mots. Les langages du politique, $\mathrm{n}^{\circ} 87$, p. 99-112.

KOMUR Greta, 2004, "L'îlot textuel et la prise de distance par le locuteur dans le genre journalistique », Le discours rapporté dans tous ses états, J. M. Munoz, S. Marnette, L. Rosier éd., Paris, L'Harmattan, p. 54-63.

KOREN Roselyne, 2001, "Quand informer, c'est dire le blanc et le noir. Quelques effets pervers de l'oscillation binaire... », Quitte ou double sens. Articles sur l'ambiguïté offerts à Ronald Landheer, P. Bogaards, J. Rooryck, P. J. Smith éd., Amsterdam, Rodopi, p. 177-200.

Koren Roselyne, RABATEL Alain, 2008, "La responsabilité collective dans la presse», Questions de communication, $\mathrm{n}^{\circ} 13, \mathrm{p} .7-24$.

KRIEG Alice, 2000, «Analyser le discours de presse. Mises au point sur le "discours de presse” comme objet de recherche », Communication, $\mathrm{n}^{\circ}$ 20, p. 75-97.

LAGAVRE Rose-Marie, 2000, «Une étrange défaite. La loi constitutionnelle sur la parité», Politix, $\mathrm{n}^{\circ}$ 51, p. 113-141.

LE BART Christian, 1999, «L'étranger comme ressourcement. Le séjour québécois d’Alain Juppé», Pôle Sud.

LÉVÊQUE Sandrine, "Crise sociale et crise journalistique. Traitement médiatique du mouvement social de décembre 1995 et transformation du travail journalistique », Réseaux, ${ }^{\circ} 17$, p. 87-117.

LUGRIN Gilles, 2001, "Le mélange des genres dans l'hyperstructure », Semen, n 13 , p. 65-96.

Maingueneau Dominique, 1987, Nouvelles tendances en analyse du discours, Paris, Hachette.

- 2007, Analyser les textes de communication, Paris, Armand Colin.

MouILLAUd Maurice, TÊTu Jean-François, 1989, Le Journal quotidien, Lyon, Presses universitaires de Lyon.

NEVEU Érik, 2000, "Le genre du journalisme. Des ambivalences de la féminisation d'une profession », Politix, nº 51, p. 179-212.

Pellat Christophe, Riegel Martin, Rıoul René, 1994, Grammaire méthodique du français, Paris, PUF.

Restier-Melleray Christiane, 2005, «Mise en proximité et politique. Les “Carnets de campagne" du Monde (19 mars-2 juin 2002)», Mots. Les langages du politique, $\mathrm{n}^{\circ} 77$, Proximité, p. 59-72.

ReY-Debove Josette, 1997 [1978], Le métalangage. Étude linguistique du discours sur le langage, Paris, Armand Colin. 
SINEAU Mariette, 1988, Des femmes en politique, Paris, Economica.

Tuomarla Ulla, 2000, La citation mode d'emploi, Helsinki, Academia Scientiarum Fennica.

- 2004, "La parole telle qu'elle s'écrit ou la voie de l'oral à l'écrit en passant par le DR», Le discours rapporté dans tous ses états, J. M. Munoz, S. Marnette, L. Rosier éd., Paris, L'Harmattan, p. 328-334.

\section{Annexe}

\section{Les séries de reportages chez les gens « ordinaires » dans la presse écrite}

\begin{tabular}{|c|c|c|c|c|c|}
\hline Journal & Titre de la série & $\begin{array}{c}\text { Dates de début } \\
\text { et de fin }\end{array}$ & $\begin{array}{c}\text { Nombre } \\
\text { d'articles }\end{array}$ & $\begin{array}{l}\text { Fréquence } \\
\text { de la série }\end{array}$ & Rubrique \\
\hline 20 Minutes & $\begin{array}{l}\text { La présidentielle } \\
\text { vue par... }\end{array}$ & $\begin{array}{l}19 / 03 / 07- \\
27 / 04 / 07\end{array}$ & 19 & quotidienne & $\begin{array}{l}\text { Présidentielle (16) } \\
\text { France (3) }\end{array}$ \\
\hline Le Parisien & $\begin{array}{l}\text { La présidentielle } \\
\text { vue par les Français }\end{array}$ & $\begin{array}{l}22 / 02 / 07- \\
26 / 04 / 07\end{array}$ & 10 & $\begin{array}{l}\text { hebdomadaire } \\
\text { (jeudi) }\end{array}$ & $\begin{array}{l}\text { Le fait du jour ou } \\
\text { Politique }\end{array}$ \\
\hline \multirow[t]{2}{*}{ La Croix } & $\begin{array}{l}\text { Les Français comme } \\
\text { ils votent }\end{array}$ & $\begin{array}{l}26 / 03 / 07- \\
03 / 05 / 07\end{array}$ & 24 & quotidienne & $\begin{array}{l}\text { France } \\
\text { Élection } \\
\text { présidentielle }\end{array}$ \\
\hline & Vu de Drocourt & $23-27 / 04 / 07$ & 5 & quotidienne & $\begin{array}{l}\text { Élection } \\
\text { présidentielle }\end{array}$ \\
\hline \multirow[t]{2}{*}{ Libération } & Vu de banlieue & $\begin{array}{l}27 / 03 / 07- \\
17 / 04 / 07\end{array}$ & 4 & $\begin{array}{l}\text { hebdomadaire } \\
\text { (mardi) }\end{array}$ & Présidentielle \\
\hline & Vu de... & $\begin{array}{l}12 / 01 / 07- \\
13 / 04 / 07\end{array}$ & 13 & $\begin{array}{l}\text { hebdomadaire } \\
\text { (vendredi) }\end{array}$ & Grand Angle \\
\hline \multirow[t]{3}{*}{ Le Monde } & $\begin{array}{l}\text { Avant } \\
\text { la présidentielle } \\
\text { La présidentielle } \\
\text { vue de... } \\
\text { puis } \\
\text { La présidentielle } \\
\text { vue de mon/ma... }\end{array}$ & $\begin{array}{l}09 / 12 / 06- \\
14 / 04 / 07\end{array}$ & 18 & $\begin{array}{l}\text { hebdomadaire } \\
\text { (samedi) }\end{array}$ & $\begin{array}{l}\text { Horizons } \\
\text { Décryptage } \\
\text { Reportage }\end{array}$ \\
\hline & Visages & $\begin{array}{l}16 / 03 / 07- \\
18 / 04 / 07\end{array}$ & 7 & irrégulière & Politique \\
\hline & J'hésite & $\begin{array}{l}12 / 04 / 07- \\
26 / 04 / 07\end{array}$ & 7 & irrégulière & Politique \\
\hline Le Figaro & Profession électeur & $13-17 / 03 / 07$ & 5 & quotidienne & France/Politique \\
\hline
\end{tabular}

